\section{Prolonged eggshell thinning caused by DDE in the duck}

Although eggshell thinning induced by $2,2-b i s-(p$-chlorophenyl)-1,1-dichloroethylene (DDE)- has been studied in many species (see review by Cooke ${ }^{1}$ ), the long term effects of this pesticide have not been investigated. Its duration of action is of great environmental significance since it will determine the effect of any pesticide ingested before the breeding season. In this respect, the finding of continuing high levels of DDE and thin eggshells in the Alaskan peregrine falcon (Falco peregrinus) in areas free from direct spraying ${ }^{2}$ argues for a prolonged effect, but direct controlled studies have been lacking. In fact, $18 \mathrm{~d}$ is the longest period to date during which thinning has been monitored (in mallards given single oral doses of $500-2,000 \mathrm{mg}$ DDE kg ${ }^{-1}$ ) and little recovery of shell thickness was observed ${ }^{3}$.

We report here the changes in eggshell thickness in the white Peking duck (a domesticated mallard) during 27 weeks following a brief exposure to dietary DDE. Ducks approximately 6 months old were divided into experimental and control flocks (three birds in each) and maintained as before ${ }^{4}$. The experimental flock was fed 250 p.p.m. DDE for $10 \mathrm{~d}$ and then control duck feed (provided by Agway Inc.) for the rest of the experiment. A rough calculation from the rate of food consumption shows that experimental ducks (body weight $5-6 \mathrm{~kg}$ ) ingested about $0.5 \mathrm{~g}$ DDE during the $10 \mathrm{~d}$. Approximately 2 months later, when all birds began laying, eggs were collected daily and frozen, and the shells were cut at the equator, that is, approximately midway between the ends. Eggshells were washed in warm water and dried overnight at $45^{\circ} \mathrm{C}$. Twenty measurements of shell thickness, ten per half, were made near the equator of the egg using an Ames micrometer. Each bird produced an average of three or four eggs per week, except for a moulting period of about 7 weeks, when none was laid (weeks 14-20, Fig. 1). As Fig. 1 shows, eggshells from ducks fed DDE were approximately $20 \%$ thinner than controls, which agrees with the maximum thinning

Fig. 1 Eggshell thickness as function of time for control $(O)$ and DDE-fed (O) white Peking ducks. Experimental birds were fed 250 p.p.m. DDE only during the time indicated. Each point represents the mean thickness for all eggs collected during the 1 week (approximately 11 eggs per flock per week). Variability, when large enough, is shown as standard error bars. Also shown are DDE residues in randomly selected egg yolks from ducks fed DDE. Values are given as p.p.m. dry weight in individual egg yolks. (These values can be converted to p.p.m. wet weight by multiplying by 0.23 or to p.p.m. lipid weight by multiplying by 1.7.$)$

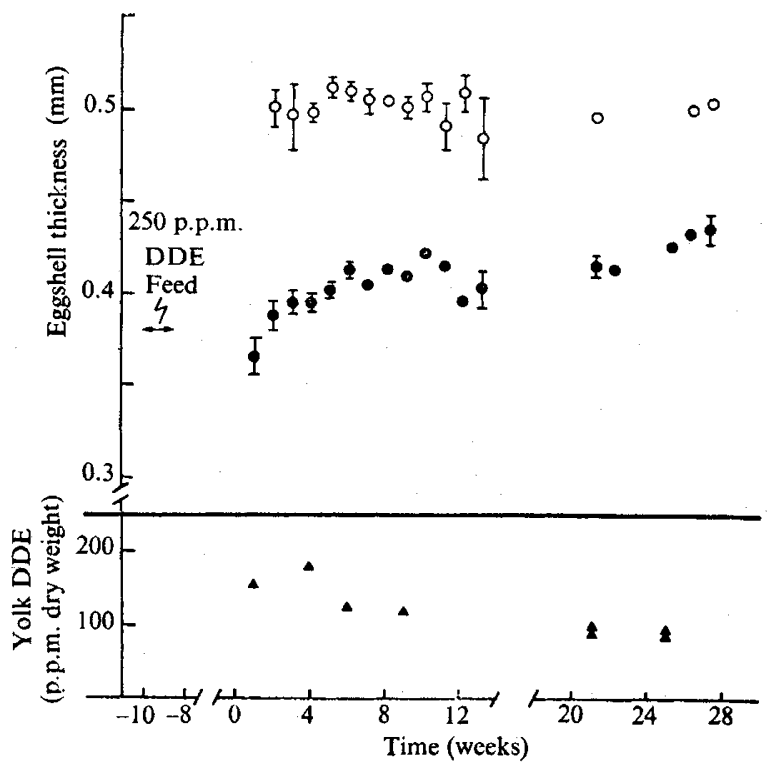

reported before 4 . Recovery of shell thickness was slow, being less than half-way at the end of 27 weeks. DDE residues in the yolks were analysed as previously described ${ }^{5}$. Initially, levels were greater than 150 p.p.m. dry weight (Fig. 1) and after 27 weeks, residue values had declined only to about 90 p.p.m. In contrast, levels of DDE residues in a control egg yolk (from week 5) were 0.23 p.p.m. Based on the values of residues shown in Fig. 1, we have calculated the approximate amount of DDE lost by a bird through its eggs. Using a value of $150 \mu \mathrm{g}$ DDE per $g$ of dried yolk, and assuming that 75 eggs were laid during the 27 weeks, each containing $18 \mathrm{~g}$ of yolk (dried weight), roughly $0.2 \mathrm{~g} \mathrm{DDE}$ or $40 \%$ of the original dose would be lost. This calculated clearance parallels the measured decrease in residue levels, that is, approximately $40 \%$ decrease in yolk DDE in 25 weeks (Fig. 1). Likewise, Tucker and Haegele ${ }^{6}$ found significant loss of DDT through the eggs of mallards. Thus, in the duck, the egg is the major route of excretion for DDE. Naturally, in the wild, the loss would be much less since many fewer eggs are laid.

Earlier work on the white Peking duck demonstrated the rapid onset of eggshell thinning, which is essentially complete within $4 \mathrm{~d}$ of exposure to 40 p.p.m. of DDE in the diet ${ }^{4}$. Our study now shows that although the egg is a major route of excretion of DDE, loss through this mechanism is relatively slow, as is recovery of eggshell thickness. Thus, DDE-induced eggshell thinning has a rapid onset and is essentially irreversible.

This study was supported by the US Public Health Service and the US National Science Foundation.

Cornell University,

Ithaca, New York 14850

\section{David B. Peakall}

David S. Miller
William B. Kinter

Mount Desert Island Biological Laboratory,

Salsbury Cove, Maine 04672

Received November 11, 1974; revised February 10, 1975.

1 Cooke, A. S., Environ. Pollution, 4, 85-152 (1973).

2 Peakall, D. B., Cade, T. J., White, C. M., and Haugh, J., Pesticide Monitoring,

J. (in the press).
3 Haegele, M. A., and Tucker, R. K., Bull. environ. Contamin. Toxicol., 11, 98-102

4 Peakall, D. B., Lincer, J. L., Risebrough, R. W., Pritchard, J. B., and Kinter, W. B., Comp. gen. Pharmacol., 4, 305-31 3 (1973).

5 Cade, T. J., Lincer, J. L., White, C. M., Roseneau, D. G., and Swartz, L. G.,

Science, 172, $955-957$ (1971).
6 Tucker, R. K., and Haegele, M. A., Bull. environ. Contamin. Toxicol., 5, 191-149 (1970).

\section{Acclimation in arctic lichens}

Using a new gas exchange method, we have followed the seasonal response of net assimilation rate (NAR) in Arctic lichens and have found rapid acclimation to temperature, light and thallus moisture content. This suggests that these organisms can adjust reversibly their patterns of physiological response to suit the atmosphere of their rather stressful habitats.

Although there is increasing evidence of temperature acclimation of net assimilation rate (NAR) in various plants, both in the field ${ }^{1-3}$ and as responses induced under laboratory conditions $^{4,5}$, investigations with higher plant systems are complicated by a combination of acclimatory response with variations of NAR resulting from seasonal production of new shoots, flowering and senescence of leaves. Lichens do not undergo seasonal growth of this sort and thus are an excellent system in which to study acclimation.

Infrared gas analysis can be used to measure NAR of plant tissue as a change in $\mathrm{CO}_{2}$ concentration within a flowing gas stream before and after it passes over the experimental plant material, which is enclosed in a special assimilation chamber. We have found that the gas flow networks and environmental control systems which are necessary are too costly and complex to permit use of more than one or two assimilation chambers. Since only one replicate sample can be included in the chamber at once, any adequately replicated experiment is very time 